\title{
BMJ Open Trends in the prevalences of congenital anomalies and age at motherhood in a southern European region: a population-based study
}

\author{
K Cambra, ${ }^{1}$ B Ibañez, ${ }^{1}$ D Urzelai, ${ }^{2}$ I Portillo, ${ }^{3}$ I Montoya, ${ }^{3}$ S Esnaola, ${ }^{2}$ F B Cirarda ${ }^{2}$
}

To cite: Cambra K, Ibañez B, Urzelai $\mathrm{D}$, et al. Trends in the prevalences of congenital anomalies and age at motherhood

in a southern European region: a population-based study. BMJ Open 2014;4: e004244. doi:10.1136/ bmjopen-2013-004244

- Prepublication history for this paper is available online. To view these files please visit the journal online (http://dx.doi.org/10.1136/ bmjopen-2013-004244).

Received 18 October 2013 Revised 5 February 2014 Accepted 10 February 2014

CrossMark

For numbered affiliations see end of article.

Correspondence to Dr Koldo Cambra; ki.cambra.contin@navarra.es

\section{ABSTRACT}

Objectives: To estimate the prevalences of the main groups of congenital anomalies and to assess their trend over time.

Design: Population-based study of prevalences. Setting: The Basque Country, Spain.

Participants: All births and all congenital anomalies diagnosed prenatally, at birth or during the first year of age, in all hospitals of the country, from 1999 to 2008.

Main outcomes measures: Total diagnosed prevalences and prevalences at birth of all chromosomal and non-chromosomal anomalies, Down's syndrome, anomalies of the nervous system, urinary, limbs, digestive system and congenital heart defects.

Results: Mean age (SD) of women at childbirth and the proportion of them over 35 years of age shifted from 32.1 (4.5) years, with $18.3 \%$ in 1999-2001, to 32.3 (4.7) years, with $23.9 \%$ in 2006-2008. Between 1999 and 2008, 991 cases of chromosomal anomalies and 3090 of nonchromosomal anomalies were diagnosed, which yields, respectively, total prevalences of $5.2 \%$ and of $16.2 \%$. Among chromosomal anomalies, Down's syndrome is the most frequent $(2.9 \%)$. With marginal statistical significance, the results point at an increasing trend in total diagnosed chromosomal anomalies, but a decreasing one in prevalences at birth. Among non-chromosomal congenital anomalies, congenital heart defects are the most frequent $(5.2 \%)$ one. Rates of all nonchromosomal, urinary and limb anomalies grew during the study period, whereas those of congenital heart defects and anomalies of the digestive system did not change significantly.

Conclusions: In the Basque Country, rates of chromosomal anomalies are higher than the overall estimated prevalence in European countries, and continue to increase slightly, which may be related to the rise in maternal age. Rates of nonchromosomal anomalies are within the European frequent range of values, and the increases observed need to be checked in the following years.

\section{Strengths and limitations of this study}

- This study analyses the trend over time of the prevalences of congenital anomalies using highly reliable data from population-based registries.

- The study provides an insight into the occurring changes in maternal age, the extent of the use of antenatal diagnosis and its impact on the prevalences of congenital anomalies at birth.

- Improvement in quality and accessibility of diagnostic tests can lead to an apparent increase in prevalences.

\section{INTRODUCTION}

Congenital anomalies affect around 2\% of births in Europe. ${ }^{1}$ They account for a great part of neonatal mortality and morbidity, and may be an important source of distress for parents and families. In the majority of cases, the aetiology of congenital anomalies remains unknown. Some increasing trends have been detected by the EUROCAT in the Pan-Europe analysis for several congenital anomalies such as hypospadias, abdominal wall anomalies, gastroschisis, trisomy 18 and renal dysplasia. ${ }^{2}$ Local trends have been more frequently detected but, in general, they need further verification.

Women's age at childbirth has been on the rise in all European countries included in Eurostat during the past few decades. From 2001 to 2008 the average maternal age in EU-17 inched up from 29.5 to 30.3 years, but geographical differences remained important, with greater values in western Europe, and differences between countries of up to 5 years. Spain, in the fifth position of the European ranking in 2008, with a mean age of women at childbirth of 30.8 years, climbed to the top in 2011 with a mean age of 31.5 years. ${ }^{3}$ Changes in demographics and in the perception of risks, along with a 
generalised extension and improvement of antenatal diagnosis, have increased (in Spain and other countries) the public awareness on reproductive health issues. Improvements in antenatal diagnosis as well as in electronic medical record systems allow for more reliable population-based studies on congenital anomalies.

The Basque Country is an industrialised, urban type community, situated in northern Spain, with around 2.2 million inhabitants. Demographic changes in the past few decades have been dramatic, and are greater than in other Spanish regions. The synthetic index of fecundity (an estimation of the number of children a hypothetical woman would have at the end of her fertile life if her fecundity corresponded to the age-specific fecundity rates of the population) fell from 2.67 in 1975 to 1.24 in 1985, 0.97 in 1990 and 1.03 in 2000, while the average of maternity age rose from 28.6 to 29.1, 30.0, 31.3 and 32.4 years in the same calendar years. ${ }^{4}$ Medical care and antenatal diagnosis are widely available to all pregnant women, and they are provided by the Basque Health Service to the entire population.

The detection of trends is an essential component of epidemiological surveillance of congenital anomalies, valuable for care and genetic advice planning and also for warning of environmental risk factors. Its interest actually grows in places where changes in reproductive health determinants may be occurring.

This report analyses the evolution of maternal age and prevalences of congenital anomalies in a 10-year period in the Basque Country, focusing on total and on specific subgroups of chromosomal and non-chromosomal anomalies.

\section{METHODS}

The study period was 1999-2008. We used data from the population-based Registry of Neonatal Screening and Congenital Anomalies of the Basque Country, integrated in the EUROCAT project and operating since the early 1990s. All anomalies diagnosed prenatally, at birth or during the first year of age, in all hospitals of the country are included. The registry covers affected live births, fetal births with gestational age 22 weeks or greater and terminations of pregnancy for fetal anomaly following prenatal diagnosis. The staff of the Registry routinely check Hospital Discharge Databases and the Registry of Terminations of Pregnancies in search of new cases, review medical reports of prenatal diagnosis (sonography, genetic test and pathology) and code all congenital anomalies.

We estimated the prevalences and $95 \%$ CI related to mothers with residence in the Basque Country of total chromosomal anomalies (International Classification of Diseases, 10th Revision (ICD-10), Q90-93, Q96-99), total non-chromosomal anomalies $(\mathrm{Q}$ chapter excluding chromosomal anomalies, D215, D821, D1810, P350, P351, P371) and of the following subgroups: anomalies of the nervous system (Q00-07), urinary (Q60-64,
Q794), limbs (Q65-74), digestive system (Q38-Q45, Q790), congenital heart defects (CHDs; Q20-26), and Down (Q90), Patau (Q914-917), Edward (Q910-913), Turner (Q96) and Klinefelter's (Q980-984) syndromes. Other major organ subgroups of non-chromosomal anomalies were not included in the analysis because their average prevalences, lower than 1 case per 1000 births, are too low to be assessed on a yearly basis. The cases with several non-chromosomal anomalies count as one case for calculating total or subgroup prevalences, but they can be assigned to more than one subgroup. More details about the anomalies included in each subgroup can be found elsewhere. ${ }^{1}$ We estimated total prevalence rates (congenital anomalies in liveborns, fetal deaths and induced abortions divided by the total number of births) and prevalence rates at birth (congenital anomalies in liveborns and fetal deaths divided by the total number of births). Data related to denominators (number of births per year, place of residence and age of the mother) were obtained from the Registry of Newborns of the Basque Country.

To explore the functional relationship between prevalences and time, we fitted Generalized Additive Models, as they are flexible unrestricted models that can properly reflect the true relationship between the variables. We included the annual number of cases as a Poisson response variable, the number of births (live and still) as the offset and the calendar year as an independent variable using thin plate regression splines. To further assess time trends, we fitted binomial regression models using a logarithmic link function, including the same former variables and adding an explanatory variable maternal age group (under 30, 30-34, 35-39 and over 40 years). Also, we used these age groups to conduct complementary age-stratified analyses for chromosomal anomalies.

In the case of Down's syndrome (DS), we also tested trends in the estimated prevalence at birth that would have occurred in the absence of antenatal diagnosis (and subsequent terminations), using for that diagnosed prevalences adjusted for the natural fetal loss risks reported by Savva et al. ${ }^{5}$ Adjusting for natural fetal losses is aimed at controlling the potential bias in the estimation of time trends in periods in which there is an increase in the number of prenatal diagnoses and, consequently, in the number of cases diagnosed prenatally that would not have survived to term and remained undiagnosed in the absence of prenatal diagnosis.

\section{RESULTS}

The total number of births in the period 1999-2008 was 191 171, rising from 51771 in the period 1999-2001 to 62329 in 2006-2008. Concurrently, age of motherhood increased (figure 1). The mean age of women at childbirth and the proportion of them with age over 35 years shifted from 32.1 years, with $18.3 \%$ in $1999-2001$, to 32.3 years, with $23.9 \%$ in 2006-2008 (table 1 ). This shift is greater among chromosomal anomalies cases, with an 


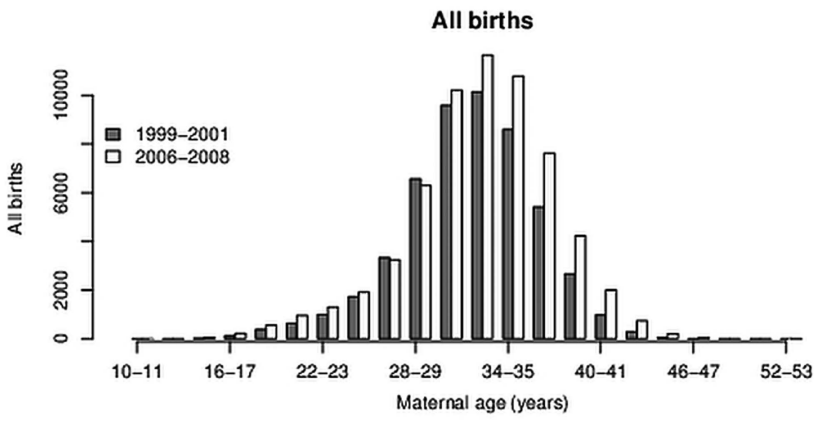

Diagnosed non-chromosomal anomalies

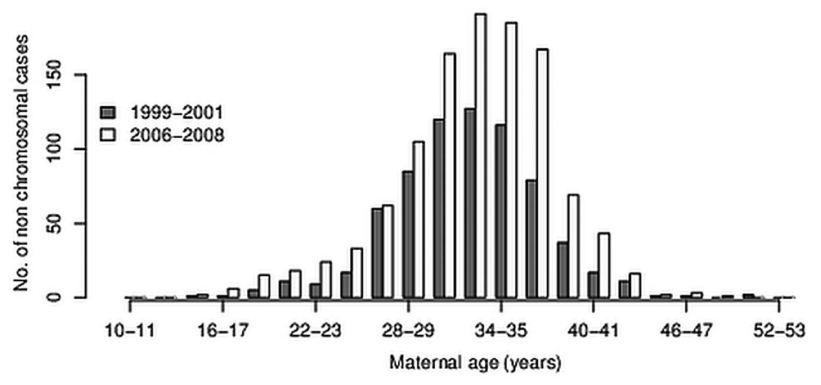

Diagnosed chromosomal anomalies

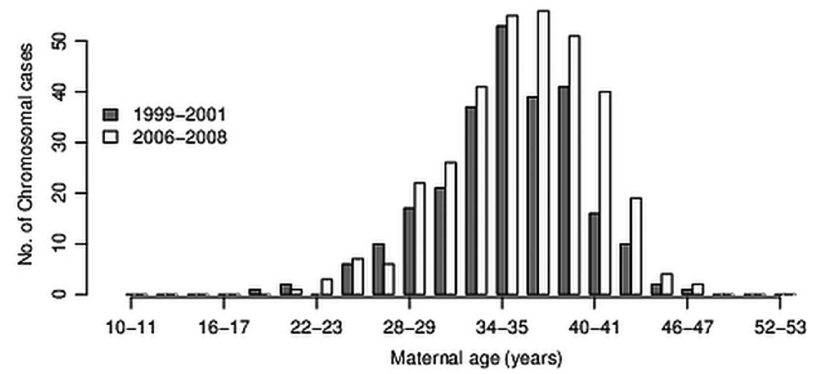

Figure 1 Age of women at childbirth and at diagnosis of a chromosomal anomaly in the Basque Country (Spain), in 1999-2001 and 2006-2008.

increase of 0.7 years in the mean age and an increase of $9 \%$ in the proportion of mothers above 35 years of age. Among cases of non-chromosomal anomalies, maternal age indicators are similar to those of all births.

Between 1999 and 2008, 991 cases of chromosomal anomalies and 3090 cases of non-chromosomal anomalies were diagnosed, which yields, respectively, prevalences of 5.18 and 16.16 per 1000 births (table 2). Diagnosis was antenatal in $83 \%$ of chromosomal anomalies and in $43 \%$ of non-chromosomal anomalies.
Among chromosomal anomalies, DS is the most frequent, accounting for $55 \%$ of all diagnosed chromosomal anomalies. The total prevalence of DS is $2.87 \%$, the estimated prevalence at birth adjusted for natural fetal losses is $2.27 \%$ and the actual prevalence of DS at birth is $0.73 \%$. Other chromosomal syndromes are much less frequent. From 1999 to 2008, Edward syndromes diagnosed were 121, Turner syndromes 81, Klinefelter 52 and Patau 42, resulting, respectively, in 0.63 ( $95 \%$ CI 0.52 to 0.75 ), 0.42 (95\% CI 0.33 to 0.52 ), 0.27 (95\% CI 0.20 to 0.35 ) and 0.22 (95\% CI 0.15 to $0.29)$ diagnosed cases per 1000 births.

The results point at certain increasing trend in total chromosomal anomalies and certain decreasing trend in prevalences at birth (table 2 and figure 2). For DS, we found, with marginal statistical significance, annual relative increases in the total prevalence of around 3\% $(p=0.079)$ and annual relative reductions of $5 \%$ in the prevalence at birth $(\mathrm{p}=0.098)$. When adjusting regression models for maternal age, increasing trends in diagnosed chromosomal anomalies disappear but decreasing trends in prevalences at birth remain unchanged, for all chromosomal anomalies and for DS. In the age-stratified analysis, the decreasing trend in the prevalence of chromosomal anomalies at birth is more clearly observed in women between 30 and 34 years of age $(p=0.031)$. Maternal age is strongly associated with the prevalence of chromosomal anomalies $(p<0.001)$, with prevalence rates 1.3, 3.2 and 8.3 times greater in women of 30-34, 35-39 and over 40 years than in women under 30 years, respectively.

Among non-chromosomal anomalies, CHDs are the most frequent $(5.0 \% 0)$, followed by urinary $(3.5 \% 0)$, nervous system (2.4\%o) and limb anomalies (2.4\%o). Prevalences at birth are around $80 \%$ of total diagnosed prevalences, with the exception of the subgroup of nervous system anomalies, for which the prevalence at birth $(0.9 \% o)$ is one-third of the total diagnosed one.

Prevalences of all non-chromosomal, urinary and limb anomalies grew during the study period with different time patterns (figure 3). Deviations from linearity are negligible in all studied subgroups of anomalies, except for the urinary subgroup, which increased between 1999 and 2003 and then plateaued, and for limb anomalies which sharply mounted from 2004 to 2008. The estimated annual relative increment of prevalence rates of all non-chromosomal anomalies is $3 \%$ and that of

Table 1 Mean age of women at childbirth and proportion of them above 35 and 40 years, in all births and in cases of congenital anomalies of the Basque Country, in 1999-2001 and 2006-2008

\begin{tabular}{|c|c|c|c|c|c|c|}
\hline & \multicolumn{3}{|l|}{ 1999-2001 } & \multicolumn{3}{|l|}{$2006-2008$} \\
\hline & Mean (SD) & $>35$ years $(\%)$ & $>40$ years $(\%)$ & Mean (SD) & $>35$ years $(\%)$ & $>40$ years $(\%)$ \\
\hline All births & $32.1(4.5)$ & 18.3 & 1.4 & $32.3(4.7)$ & 23.9 & 2.9 \\
\hline Chromosomal CA & $34.6(4.5)$ & 42.6 & 7.4 & $35.3(5.0)$ & 51.5 & 12.0 \\
\hline Non-chromosomal CA & $32.1(4.6)$ & 21.1 & 3.6 & $32.3(5.1)$ & 27.2 & 3.2 \\
\hline
\end{tabular}


Table 2 Trends in congenital anomalies in the Basque Country (Spain), from 1999 to 2008

Prevalences (cases/1000 births)

n

Total diagnosed anomalies

Chromosomal

DS

usted fetal loss

Non-chromosomal

All

Nervous system

Urinary

Digestive

Limbs

Heart

Anomalies at birth

Chromosomal

All

DS

Non-chromosomal

All

Nervous system

Urinary

Digestive

Limbs

Heart

DS, Down's syndrome.

$3090 \quad 16.16$ (15.61 to 16.74

2.38 (2.17 to 2.62 )

3.50 (3.25 to 3.78$)$

1.59 (1.42 to 1.78$)$

$460 \quad 2.41$ (2.19 to 2.64 )

$962 \quad 5.03$ (4.72 to 5.36 )

$0.73(0.61$ to 0.86$)$

1.44 (1.28 to 1.63$)$
1999-2001

2006-2008

4.95 (4.37 to 5.60$)$

2.76 (2.34 to 3.26$)$

2.24 (1.86 to 2.70 )

13.75 (12.78 to 14.80$)$

1.99 (1.63 to 2.42 )

2.41 (2.02 to 2.89 )

1.31 (1.03 to 1.68 )

$1.62(1.30$ to 2.02$)$

4.87 (4.29 to 5.51$)$

5.36 (4.81 to 5.97$)$

3.10 (2.68 to 3.57$)$

2.46 (2.09 to 2.88 )

17.76 (16.75 to 18.84$)$

2.84 (2.44 to 3.30$)$

3.64 (3.19 to 4.16$)$

1.83 (1.52 to 2.21$)$

4.09 (3.61 to 4.63 )

4.81 (4.29 to 5.40 )

1.49 (1.18 to 1.87$)$

1.02 (0.77 to 1.35$)$

$1.16(0.91$ to 1.46$)$

0.66 (0.48 to 0.90$)$

0.97 (0.93 to 1.01$)$

0.95 (0.9 to 1.01 )

1.01 (0.99 to 1.04$)$

1.03 (1.00 to 1.06$)$

1.02 (0.99 to 1.06$)$

0.174

0.079

$1.00(0.98$ to 1.02$)$

$0.161 \quad 1.00(0.97$ to 1.04$)$

0.878

0.669

$1.04(1.02$ to 1.05$)<0.001$

1.05 (1.01 to 1.08$)$

1.04 (1.02 to 1.07$)$

0.007

$1.04(1.03$ to 1.05$)<0.001$

1.05 (1.01 to 1.08$)$

0.007

1.03 (0.99 to 1.08$)$

0.002

1.04 (1.01 to 1.07$)$

0.003

1.03 (0.99 to 1.08$) \quad 0.105$

$1.17(1.13$ to 1.21$)<0.001$

1.01 (0.98 to 1.03$) \quad 0.619$

10.72 (9.86 to 11.65$)$

14.07 (13.17 to 15.03$)$

1.17 (0.92 to 1.48$)$

1.04 (1.03 to 1.06$)$

0.64 (0.45 to 0.91$)$

3.11 (2.70 to 3.59$)$

1.10 (1.04 to 1.16$)$

1.05 (1.02 to 1.09$)$

1.03 (0.99 to 1.08$)$

$1.17(1.13$ to 1.22$)$

$3.23(2.80$ to 3.71$)$

1.20 (0.93 to 1.55$)$

$1.33(1.04$ to 1.70$)$

3.23 (2.80 to 3.71 )

0.98 (0.96 to 1.01$)$

0.876

0.619


Figure 2 Estimated prevalences in each calendar year (error bars) and Generalised Additive Models fits (lines) of chromosomal congenital anomalies in the Basque Country (Spain), from 1999 to 2008.
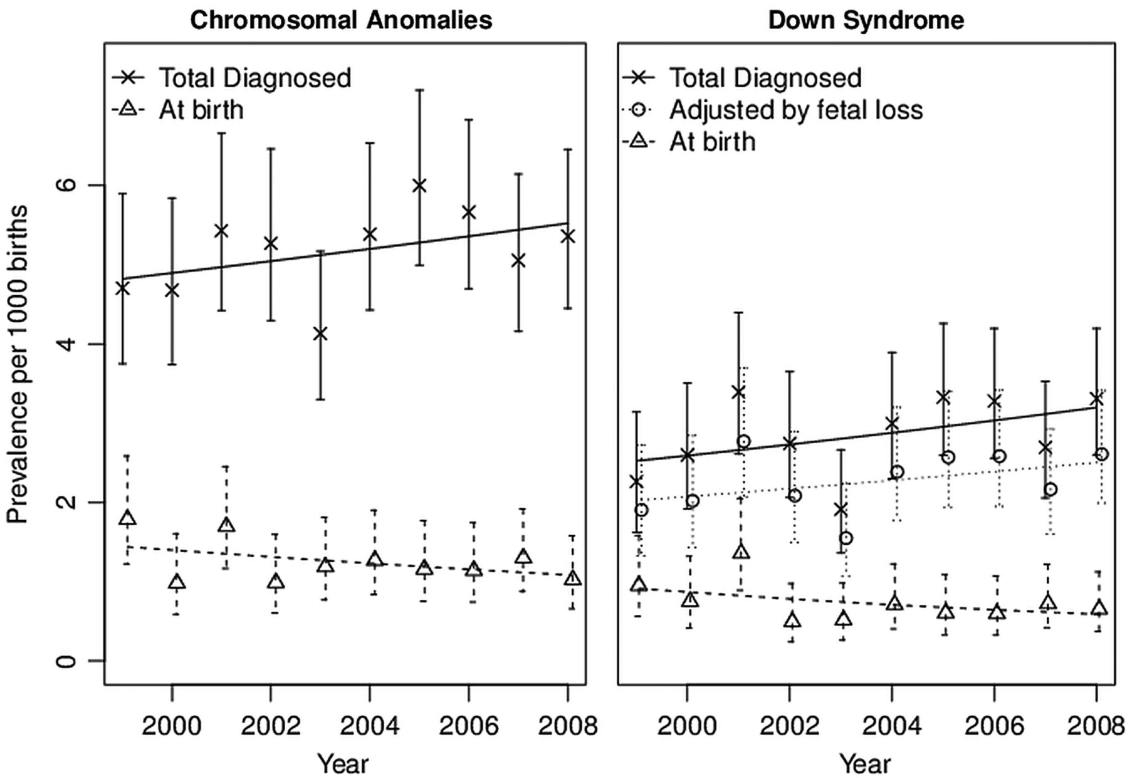

nervous system anomalies is $5 \%$. When models are adjusted for maternal age, trend estimates and statistical significances remain unchanged (table 2). With respect to mothers under 30 years, prevalence rates of all nonchromosomal anomalies were 0.93 (95\% CI 0.85 to 1.02) for mothers between 30 and 34 years, 1.0 (95\% CI 0.90 to 1.10 ) for mothers between 35 and 39 years, and 1.30 (95\% CI 1.06 to 1.51$)$ for those aged over 40 years.

\section{DISCUSSION}

During 1999-2008, the age of women at childbirth shifted towards later ages, and there was an increase in diagnosed prevalences of chromosomal and nonchromosomal anomalies in the Basque Country. Among non-chromosomal anomalies, the evolution of prevalences is particular of each subgroup, with no common pattern.

Data used in this study were obtained from the Registry of Neonatal Screening and Congenital Anomalies of the Basque Country. The registry follows the EUROCAT project guidelines and it was launched several years before the initial date of this project. As a consequence, the data are highly reliable and have improved comparability with the results from other registries. ${ }^{6}$ Surveillance of the evolution of congenital anomalies conceivably allows detecting changes in risk that may occur locally or on a greater scale. However, antenatal diagnosis leads to detect more non-viable cases that otherwise would have been miscarriages and remain undiagnosed, and, therefore, improvements in quality and accessibility of diagnostic test can make the estimation of trends in congenital anomalies rates more prone to bias than in other health outcomes. This is an important issue to take into account when interpreting changes in rates of diagnosed congenital anomalies.

Prevalence of chromosomal anomalies in the Basque Country is higher than the overall estimated prevalence for Europe, $5.2 \%$ vs $3.8 \%$ in all chromosomal anomalies, and $2.9 \%$ vs $2.1 \%$ in the case of $\mathrm{DS}^{2}{ }^{7}$ It is well known that older mothers are at higher risk of chromosomal anomalies, and that risk increases exponentially from the age of 30, not only for DS, but also for other less prevalent chromosomal syndromes, such as trisomies 13 and $18 .^{8}$ The high maternal age of Basque mothers, in fact, at the top of the European ranking, can explain why prevalences of chromosomal anomalies are higher in the Basque Country. Likewise, the increase in maternal age from 1999 to 2008 would be the primary reason to explain the observed positive trend in all chromosomal anomalies and DS prevalences. Our findings support this view, as trends are no longer statistically significant when adjusting for maternal age. A secondary explanation, for trends and for the differences with other European regions, may be differences in case ascertainment derived from the increasingly frequent use of prenatal diagnoses in the Basque Country, which, in turn, is partially provoked by the rise in maternal age. Increases in prevalences of chromosomal anomalies have also been reported in other European regions. ${ }^{2}$

Prevalences at birth of chromosomal anomalies show certain decreasing trend, which is related to the extension and use of antenatal diagnosis and the decision that most of women make after receiving a positive diagnosis. On average, $80 \%$ of all chromosomal anomalies were diagnosed antenatally, $74 \%$ in 1999-2001 and $85 \%$ in 2006-2008. In the Basque Country, 90\% of the women who received an antenatal diagnosis of chromosomal anomaly decided to terminate the pregnancy. This proportion is similar to that found in England and Wales where, as Morris and Alberman ${ }^{9}$ reported, dramatic changes in demography have been offset by improved antenatal diagnosis and have resulted in no substantial changes in birth prevalences of DS.

The prevalence of non-chromosomal congenital anomalies in the Basque Country, contrary to 
Figure 3 Estimated prevalences in each calendar year (error bars) and Generalised Additive Models fits (lines) of non-chromosomal congenital anomalies in the Basque Country (Spain), from 1999 to 2008.
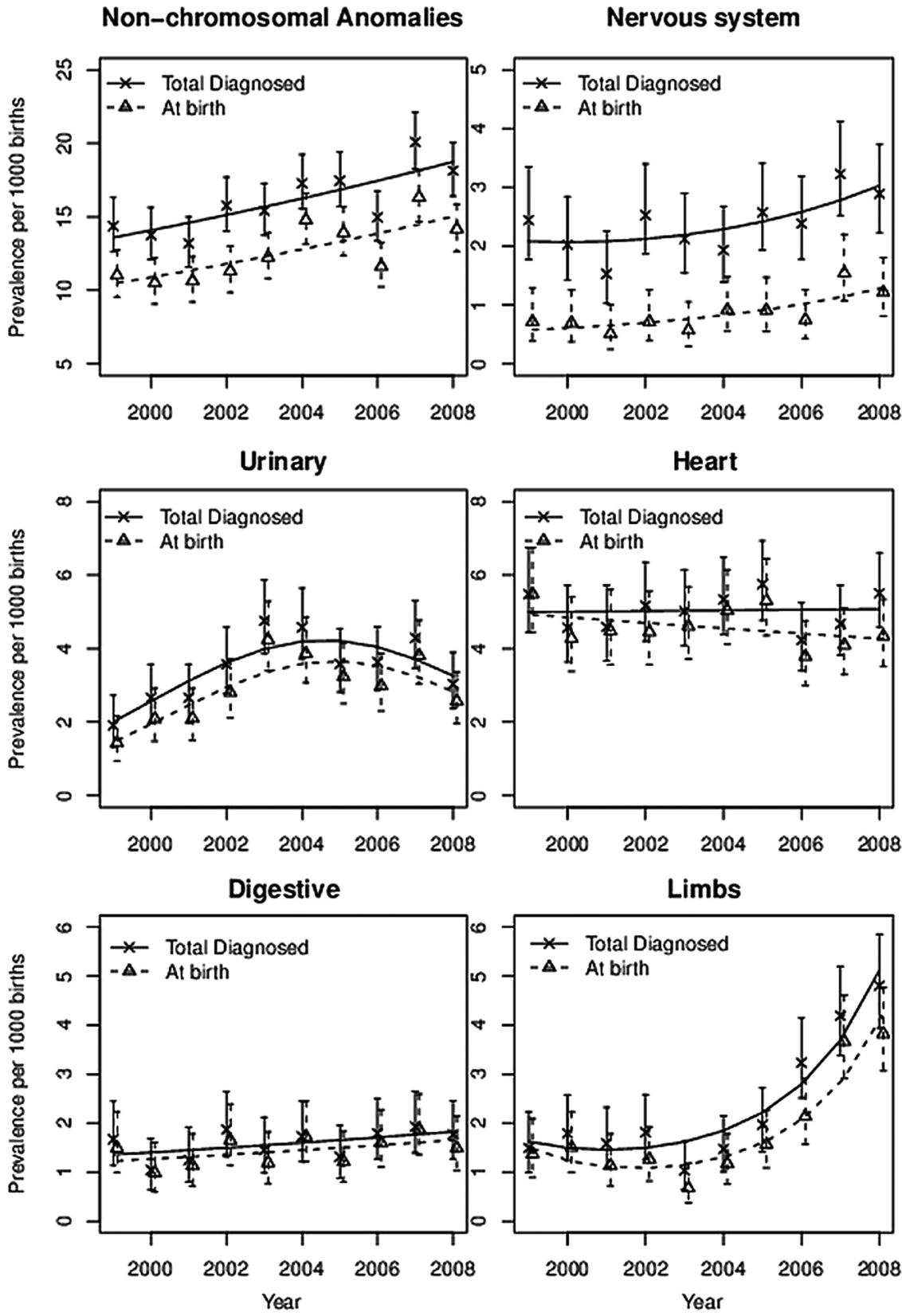

chromosomal anomalies, is lower than overall rates reported in Europe, $12.8 \%$ ov $20.2 \%$ o. ${ }^{2}$ By subgroups, those of the nervous system, digestive and urinary anomalies, with deviations smaller than $10 \%$, can be considered similar to the overall European rate; CHD rates are $20 \%$ lower in the Basque Country.

The relationship between maternal age and the prevalence of some subgroups of non-chromosomal anomalies has been reported to be U-curves or J-curves, with younger and older mothers showing an increased risk. ${ }^{10-12}$ Our findings for total non-chromosomal anomalies, with the lowest prevalence in mothers aged between 30 and 34 years, are in agreement with this type of relationship. Loane et $a l^{13}$ performed a populationbased prevalence study, covering a total of 1.75 million births from 2000 to 2004, and they found teenage mothers to have the highest prevalence of all non- chromosomal congenital anomalies. The prevalence decreased as the maternal age increased, until a slight increase was observed in mothers with 40-44 years of age and a further increase in mothers above 45 years. They also reported that the maternal age pattern of risk differs between countries, and concluded that, unlike chromosomal anomalies, it is not biological age that is associated with risk of non-chromosomal congenital anomalies, but reproductive, social and ethnic factors and exposure of lifestyle factors that have a different relationship with maternal age in different countries. They concluded that the increase of risk due to maternal age can be considered negligible, especially when compared with chromosomal anomalies. Association of paternal age with risk of certain birth defects has been reported for younger paternal ages, also with relatively low increases in risk as age grows. ${ }^{14}$ 
All non-chromosomal anomalies increased during the study period, but this result needs to be treated with caution. All non-chromosomal anomalies are very broad and heterogeneous, with different aetiologies that can hardly be related to a common true change in risk. Besides, in two of the subgroups, limbs and urinary anomalies, improvements in case ascertainment cannot be completely ruled out, as prevalences before the observed sudden rise were clearly below the average prevalences reported from other registries.

We did not find any change in CHD prevalences, in line with the trends observed in some parts of Europe, where after an increase in the early 1990s due to improvements in echocardiographic investigations, rates have remained stable. $^{2} 15$ Prevalences of congenital anomalies of nervous and digestive systems show a slight steady increase from 1999 to 2008. Decreasing trends in neural tube defects (NTDs) have been reported in some parts of Europe, ${ }^{2}$ but in other places no significant decrease has been found. ${ }^{16} 17$ NTD risk has been associated with socioeconomic status. This has raised the issue whether periconceptual folic acid recommendations are being followed. Spanish data about folic supplementation in pregnancies, collected from the INMA project, point at extensive compliance of recommendations in the third month of pregnancy (94\% of women), lower compliance in the second month of pregnancy $(66 \%$ of women) and a scarce preconceptual compliance (19\%), suggesting that folic acid supplementation in Spain is still an area of improvement for NTD reductions. ${ }^{18}$

Difference between diagnosed anomalies and prevalences at birth is slight for digestive, limbs and urinary anomalies, while it is notable for the nervous system, reflecting differences in severity and in the proportion of antenatal diagnosis, much more frequent for anomalies of the nervous system (in $82 \%$ of the diagnosed cases) than in limbs $(57 \%)$, digestive $(28 \%)$ and urinary anomalies $(22 \%)$.

Maintaining the surveillance of non-chromosomal anomalies is essential. It is necessary to check whether the observed trends continue or disappear in the following years, and complementary data analyses are needed in order to assess whether, associated or not with the increasing trends, there is spatial clustering of cases that can share the same aetiology.

\section{CONCLUSION}

In the Basque Country, chromosomal anomalies rates are high and continue to increase slightly, which may be related to the rise in the maternal age. Rates of nonchromosomal anomalies are within the European frequent range of values, and the increases observed in some of them need to be checked in the following years.

\section{Author affiliations}

${ }^{1}$ Navarrabiomed-Fundación Miguel Servet-Red de Investigación en Servicios de Salud en Enfermedades Crónicas (REDISSEC), Pamplona, Spain ${ }^{2}$ Departamento de Salud, Gobierno Vasco, Vitoria-Gasteiz, Araba, Spain ${ }^{3}$ Osakidetza-Servicio Vasco de Salud, Vitoria-Gasteiz, Araba, Spain
Contributors KC designed the project, checked the data, interpreted the results and drafted and revised the manuscript. BI participated in the study design, conducted the statistical analysis, interpreted the results and reviewed the manuscript. FBC, DU and IP participated in the study design, collected the data, created the databases and reviewed the manuscript. SE and IM took part in the study design and reviewed the manuscript.

Funding This study was partially funded by the Instituto de Salud Carlos III (FIS PI 08/1392)) of the Spanish Ministry of Economy and Competitiveness.

Competing interests None.

Ethics approval Clinical Research Ethics Committee of the Basque Country.

Provenance and peer review Not commissioned; externally peer reviewed.

Data sharing statement No additional data are available.

Open Access This is an Open Access article distributed in accordance with the Creative Commons Attribution Non Commercial (CC BY-NC 3.0) license, which permits others to distribute, remix, adapt, build upon this work noncommercially, and license their derivative works on different terms, provided the original work is properly cited and the use is non-commercial. See: http:// creativecommons.org/licenses/by-nc/3.0/

\section{REFERENCES}

1. Boyd PA, Haeusler M, Barisic I, et al. Paper 1: the EUROCAT network-organization and processes. Birth Defects Res A Clin Mol Teratol 2011;91(Suppl 1):S2-15.

2. Loane M, Dolk H, Kelly A, et al. Paper 4: EUROCAT statistical monitoring: identification and investigation of ten year trends of congenital anomalies in Europe. Birth Defects Res A Clin Mol Teratol 2011;91(Suppl 1):S31-43.

3. EUROSTAT. Mean age of women at childbirth (code: tps00017). http://goo.gl/8Lzfk (accessed 4 Jun 2013).

4. EUSTAT. Synthetic index of fecundity and average maternity age according to year. http://goo.gl/jlBXf (accessed 4 Jun 2013).

5. Savva GM, Morris JK, Mutton DE, et al. Maternal age-specific fetal loss rates in Down syndrome pregnancies. Prenat Diagn 2006;26:499-504

6. Loane M, Dolk H, Garne E, et al. Paper 3: EUROCAT data quality indicators for population-based registries of congenital anomalies. Birth Defects Res A Clin Mol Teratol 2011;91(Suppl 1):S23-30.

7. Khoshnood B, Greenlees R, Loane M, et al. Paper 2: EUROCAT public health indicators for congenital anomalies in Europe. Birth Defects Res A Clin Mol Teratol 2011;91(Suppl 1):S16-22.

8. Savva GM, Walker K, Morris JK. The maternal age-specific live birth prevalence of trisomies 13 and 18 compared to trisomy 21 (Down syndrome). Prenat Diagn 2010;30:57-64.

9. Morris JK, Alberman E. Trends in Down's syndrome live births and antenatal diagnoses in England and Wales from 1989 to 2008: analysis of data from the National Down Syndrome Cytogenetic Register. BMJ 2009;339:b3794.

10. Reefhuis J, Honein MA. Maternal age and non-chromosomal birth defects, Atlanta-1968-2000: teenager or thirty-something, who is at risk? Birth Defects Res A Clin Mol Teratol 2004;70:572-9.

11. Ooki S. Maternal age and birth defects after the use of assisted reproductive technology in Japan, 2004-2010. Int J Womens Health 2013;5:65-77.

12. Gill SK, Broussard C, Devine O, et al. Association between maternal age and birth defects of unknown etiology: United States, 19972007. Birth Defects Res A Clin Mol Teratol 2012;94:1010-18.

13. Loane M, Dolk H, Morris JK. Maternal age-specific risk of non-chromosomal anomalies. BJOG 2009;116:1111-19.

14. Green RF, Devine O, Crider KS, et al. Association of paternal age and risk for major congenital anomalies from the National Birth Defects Prevention Study, 1997 to 2004. Ann Epidemiol 2010;20:241-9.

15. Oyen N, Poulsen G, Boyd HA, et al. National time trends in congenital heart defects, Denmark, 1977-2005. Am Heart J 2009;157:467-73 e1.

16. Poretti A, Anheier T, Zimmermann R, et al. Neural tube defects in Switzerland from 2001 to 2007: are periconceptual folic acid recommendations being followed? Swiss Med Wkly 2008;138:608-13.

17. Abeywardana S, Bower C, Halliday J, et al. Prevalence of neural tube defects in Australia prior to mandatory fortification of bread-making flour with folic acid. Aust N Z J Public Health 2010;34:351-5.

18. Sanfélix-Gimeno G FI, Librero J, Peiró S. Characterization of folate supplementation in pregnancy, based on a combination of health information systems. Gac Sanit 2012;26:7. 